\title{
EXPERIMENTAL PYORRHEA.
}

\author{
By Percy R. Howe, A. B., D. D. S., Boston, Mass. \\ Assistant Professor Dental Research Harvard University. \\ Chief of Research Forsyth Dental Infirmary.
}

(Read before the National Dențal Association at Its Twenty-Second Annual Session, Chicago, Ill., August 5-9, 1918.)

I THIS paper I wish to take up with you, those experiments in which the various theories upon Pyorrhea have been put to the actual test of their ability to produce the disease.

It may be objected by some that the conditions that I shall describe are not true pyorrhea. Pyorrhea alveolaris is a loose expression, but $\mathrm{I}^{*}$ shall employ it in its broadest sense and consider as pyorrhea, lesions about the teeth that result in the destruction of their supporting tissues. This definition is meant to include all types, and all stages, of this disturbance. The attempt to refine the term "Pyorrhea," often tends to obscure the main principles that underly the trouble. This, it is most desirable to avoid.

There are certain conditions that produce in man such pronounced loss of the supporting tissues of the teeth that they need no more than be mentioned. One of these is phosphorous poisoning. Here the teeth lose their supporting structures and are destroyed. Arsenic applied to the gums destroys the tissue, resulting in loss of the teeth. Mercurical poisoning acts in the same way. These are strong chemical poisons and their effects are most marked manner upon the tissues about the teeth. The alveolar structure which is enscaffold upon the true bones of the maxilla is prompt to show such deleterious effects.

Destruction of these tissues may be brought about by a small rubber ring being allowed to work its way into these tissues. Hasty and improper regulating has been known to produce the same effect. The trauma of irregular articulation is so well known that by some it has been thought to be the sole cause of pyorrhea. Tartar by its constant accumulation becomes a foreign irritant to the tissues and acts to cause irregularity in the natural functions of the soft tissue and also to induce absorption of the alveolar process. But these are mechanical effects which disturb the circulation of the divesting tissues and which cause their disintegration. These effects are marked and self-evident and experimentation could add but little, if anything, to our understanding of the process. Thruout all the literature upon the subject of pyorrhea these are known and accepted ideas.

But there are other theories of pyorrhea that need further study. One of these is the very striking idea that oral endameba are the cause of pyorrhea and that emetin is a specific for the disturbance. (1) To one that has observed these protozoa it must be evident that the conception of their being an etiological factor in pyorrhea is certainly fascinating. These endameba have long been known, altho little previous attention had been called to them. When this theory was first evolved I took the position that oral endameba were nonparasitic and were in no way to be compared with the endameba histolytica of tropical dysentery.

Undoubtedly the originators of this 
theory had the parasitic properties of the histolytica in mind when they declared that the oral endameba were the specific cause of pyorrhea. I have found oral endameba in every description of mouths and have written fully of this elsewhere. (2) I carried out the following experiments. I established pockets about the teeth of guinea pigs and sealed in as well as possible active endameba. Only spontaneous healing occurred.

Craig (3) has done extensive work upon the endameba in man. He terms the endameba of the mouth endameba gingivalis. He says concerning them that the "size varies from seven to thirtyfive microns, the average diameter being from twelve to twenty microns; ectoplasm and endoplasm well developed, the ectoplasm being visible only when motility is present. The ectoplasm is quite refractive and movement is quite active, the pseudopodia being well developed and short and blunt, or long and finger-like in shape. The endoplasm is granular, contains very numerous food vacuoles, non-contractile, but in the living specimen a distinct nucleus is not visible in most instances. The organism is actively phagocytic and red blood corpuscles are often ingested. Reproduction apparently occurs only by simple division during the vegetative stage of development. Cysts are formed containing one neucleus, but no reproductive phenomenon have been observed. The cysts measure from eight to ten microns in diameter, altho larger cysts have been observed in the human mouth. It also occurs in a large proportion of perfectly healthy mouths and there has never been the least proof adduced, of an experimental nature, connecting endameba gingivalis with the production of pyorrhea."

I had the pleasure of examining at Paris the specimens of Dr. Chiavaro of Rome, and to find that he agreed with me in his opinion of their office in the mouth. He states that they are harm- less scavengers engaged in the auto-disinfection of the mouth. After this theory began to be generally questioned, I discussed the matter with Dr. Smith and he suggested that they might still be factors in pyorrhea thru toxin formation derived from symbiotic relationship with bacteria. There seemed to be no reason to support the idea, indeed it was highly theoretical and in the experiments upon guinea pigs there was no evidence to support it. I believe the endamebic theory has been practically given up.

The theory that pyorrhea is caused by bacterial invasion is, of course, the most prominent at the present time. That bacteria are always associated with pyorrhea is beyond question. No specific bacterium has been found, but a great variety of microbic life is pressnt. What is the nature of this bacterial flora? What is its source? Does it become parasitic, if so how does it become parisitic? Is it the first cause or is it secondary are questions upon which we need further light.

In considering this it is necessary for us to know the types of micro-organisms found in pyorrhea and something of their action in tissues Many of the reports upon the bacteriology of pyorrhea have been based upon smears. (4) The objection to be raised against smears is that many microorganisms are very pleomorphic. Indeed, all micro-organisms appear in different forms during different stages in their life cycles. They also react to different staining reactions according to the media in which they live. On the other hand cultivation upon artificial media is not without its draw-backs for certain micro-organisms grow rapidly in one culture media and not so well in another. Some do not grow at all. The living together of various types of micro-organisms, or the symbiotic relationship, is rarely represented in the culture tube. So that it is difficult to obtain an exact 
representation of what goes on in the mouth. Nevertheless, no specific bacterium has so far been found. The following microbic life has been reported. The ameba, which we have already discussed; spirochetes of various types, such as treponema mucosum, have been cultured and studied. Treponema mucosum is a mucin-former and gives the characteristic odor of pyorrhea (Noguchi.) (5) From smears other spirochetes are given, as S. refringens. S. vincenti. These Drew and Griffin (6) differentiated by the Congo-red method. Streptococci, of both the viridans and hemolytic type, many cocci, staphylococci, both albus and aureus, catarrhalis, teragenus, vibrios, fusiform and other bacilli, all have been mentioned.

In my cultural examinations, I have found spirochetes and have cultivated them in horse serum, staphylococci, streptococci, both short-chain green-producing colonies and hemolytic streptococci, bacillus bifidus, (7) colon bacillus, catarrhalis. These were cultivated aérobically and anaerobically principally on blood agar.

In thirty cases by the Avery method and by the Avery, Chickering, Cole and Dochez (8) method, I have found pneu- mococci but once. This was type IV. Goadby (9) remarks that he has found no pneumococci in pyorrhea. Others have reported the opposite. In the case containing type IV the material was placed into type $1,2,3$, anti-pneumococcus sera furnished by the Rockefeller Institute and into bile. They were soluble in bile and gave no reaction in the sera which indicates a type IV pneumococcus. The source of this material was from a very bad case of pyorrhea.

In nine other cases the material was drawn from the pyorrhea pockets by means of sterile pipette, cultured for five hours in $4 \mathrm{c}$. c. of blood broth. Cells were thrown down, then the microorganisms in the supernatant liquid decanted and thrown down, 2 c. c. sterile salt added and these were injected intraperitoneally into 15 gram mice with no result. Mice are alive and well.

In ten other cases the same proceeding was used except the cultures were incubated.

In twelve cases the material from the pyorrhea pockets was injected directly into mice, for it was thought that some of the micro-organisms that would not grow in culture media might grow in the body of the animals.

TABLE.

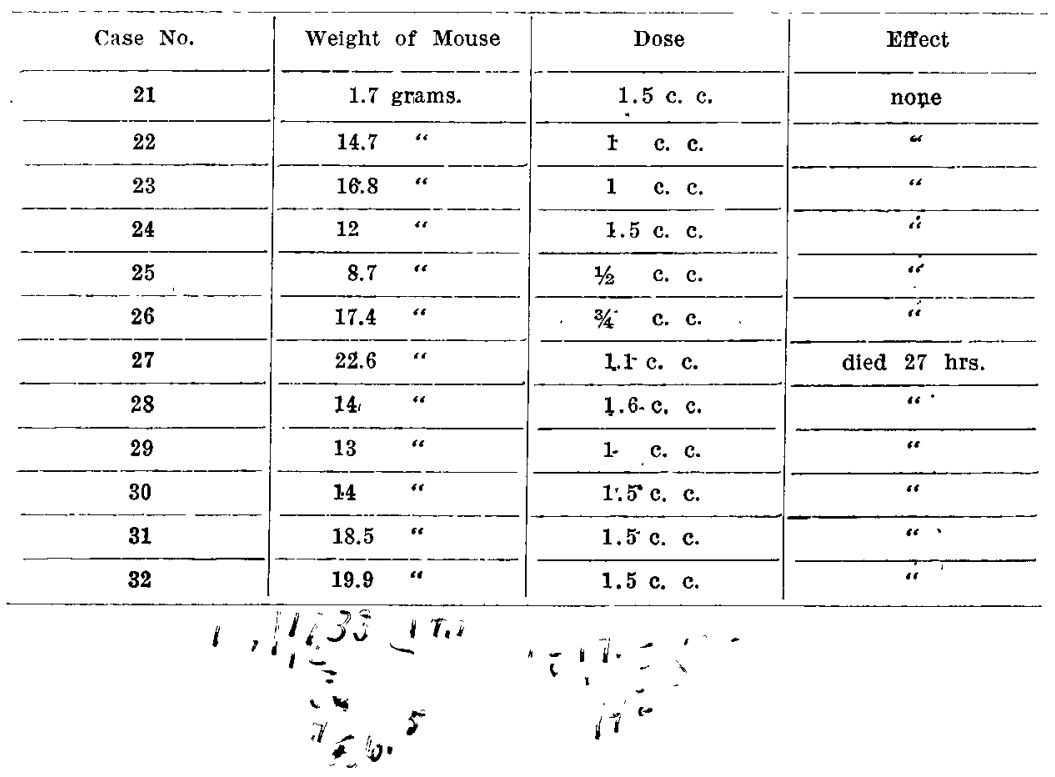


For dosage we have in this table an average of 1.2 c. c. to a 15.8 grams mouse. Only one of these mice died. Examination of the peritoneal washings showed staphylococcus infection with colon bacillus. This may have been due to intestinal puncture, but this rarely happens and we have found staphylococcus and colon bacillus together in an examination of pyorrhea pockets.

However, this shows that in the majority of cases that the virulence of the organisms of pyorrhea pockets to mice at least is low, for this dose mentioned above would amount to about five and a quarter liters injected in the case of a man of seventy kilos or of one hundred and fifty-four pounds, while in a mouse of 15 grams weight one one-millionth of a cubic centimeter of virulent streptococcus will produce septicemia and death. In injecting mice with these massive doses intraperitoneally two deaths only have occurred. One showed type IV pneumococci the other staphylococci and colon bacillus. In a colony of 15 mice, four untreated mice died. Goadby (9) on the contrary injected subcutaneously ten guinea pigs and five rabbits with an emulsion of pyorrhea pus in sterile broth. All but one guinea pig and one rabbit died. He does not give his dosage.

It is well known, however, that such micro-organisms are as a rule low in virulency. It is also known that they constitute the usual oral flora of the adult. And that it is only under certain circumstances that the tissue looses its natural refractory state toward them and is invaded.

I have injected some of these microorganisms into the tissue about the teeth of guinea pigs but have never been able to establish in this way any condition that stimulates pyorrhea. Nothing but spontaneous healing has occurred.

Dr. Hartzell (10) in his very interesting studies says, I believe, that he was never able to produce a dental abscess by injection of streptococcus viridans. So far as I know no one has yet been able to produce pyorrhea by the injection of micro-organisms, or any mixture of micro-organisms about the teeth. As regards the participation of bacteria in pyorrhea alveolaris, Miller (11) says, "Micro-organisms which possesses pyogenic properties temporarily or permanently inhabit every mouth. If, therefore, the power of resistence of the peridental tissue be impaired by any of the above mentioned local or constitutional causes in such a manner as to furnish a suitable culture medium for the bacteria. They will, of course, begin their ravages and the usual symptoms follow." That is, the tissue alteration is the first cause. The bacterial invasion is secondary. In view of what I have shown this seems to me the only possible explanation of the part played by the microbic life in this lesion. There is an enormous amount of evidence to support such a principle and so far as pyorrhea is concerned none that I know of that will support any other, viz, that the infection is secondary.

The infection must be proceeded by chemical or mechanical tissue disturbance; by trauma; by constitutional effects due to nervous, or anomalous metabolic conditions.

So far as ameba is concerned no tissue effect is to be seen. As for the spirochetes of the type under consideration Noguchi says that they will not grow in healthy tissue. He regards the etiological factor in pyorrhea as an anomalous metabolic condition, the infection as secondary. The study of the fusiform bacillus in pyorrhea and other lesions by Dick (12) and others (13) (14) show it to be secondary. And so it is with the other micro-organisms of pyorrhea so far as is known.

If the infective part of pyorrhea is a secondary matter in its pathology and if the infection consists of a mixed flora 
what must be said concerning the use of vaccines in its treatment?

The most we can expect from the use of a vaccine would be to produce an effect upon the sequelae of a previous disturbance. Vaccines as they are ordinarily prepared for pyorrhea are prepared from aerobic cultures This is one limitation, for it would only produce its effects upon the aerobic organisms of pyorrhea, while a great proportion of the micro-organisms of pyorrhea are anaerobes.

Vaccines for use in this connection are prepared by short time cultivation. Such preparations would not have any effect upon the micro-organisms that are most intimately connected with the process, if we are to judge by the effect upon the media in the culture tube. I refer to the mucin-forming and media-liquifying spirochetes for these micro-organisms require from eight to ten days or even longer for development. Vaccines might affect the staphylococci. With regard to the streptococci upon which an immense amount has been done, we can only say that much more remains to be done. The streptococci are usually classified as those that have the property of producing methemoglobin from oxyhemoglobin and those that do not. Pneumococci possess the same property. (15) This is the basis of distinction between the streptococcus viridans and the hemolytic streptococci. There are certain discrepancies in this classification as are continually being shown. Howel (16) says, "No correlation was observed between complement fixation tests and groups based on hemolysis and nonhemolysis." Vaccine and serum treatment has not been considered effective in this connection by the majority of workers upon the subject. (17) (18)

So that there seems to be little reason to feel that vaccines would be effective in pyorrhea. On the whole, then the beneficial results to be expected from vac- cines must be limited. The infection may be disposed of surgically or it may be effectively treated by injections of substances like mercury succinimid. (19)

The experimental condition that stimulates pyorrhea most closely is that brought about in guinea pigs by feeding them milk and oats or some constipating diet. In about three weeks the teeth loosen, the gums bleed and the joints are affected. In some animals the teeth have become so loose that they could be removed with an excavator. In certain cases the teeth will tighten upon giving a cathartic. This has been shown by McCollum and by Pitz (20) (21). Jackson and Moody (22) (23) and others have studied this experimental condition from a pathological and bacteriological standpoint. I have carried out this experiment on several batches of guinea pigs with special reference to this very pronounced dental effect. The guinea pig has a delicate caecum and when this becomes impacted thru an improper diet the teeth loosen and a joint affection occurs. Some have suggested that this tooth and joint condition might be an infection. However, this may be the control lies within the intestine as Pitz (21) has shown for by adding $10 \%$ to $20 \%$ lactose to the diet of grains, the guinea pigs improve. It prevents the formation of such intestinal putrefactive substances as, indole, scatole and phenol. He attributes the condition to the pasty feces of the oat diet and to the resulting toxemia. This condition is known as experimental scurvy and its study presents some most important dietetic material for the student of dental conditions. Upon improper diets the laboratory animals become the victim of many low grade infections. Indeed, Pemberton (22) says, "that cases showing unmistakable and obvious sources of infection in teeth, tonsils, or elsewhere may improve or recover completely upon a dietary regime. 
The character of foods in the control of dental disturbance is undoubtedly a matter of great importance. Particularly in the growing child. This experimental pyorrhea is only one of the noticeable effects. McCollum (21) and Mendel and Osborne (23) have shown how it is possible to check or to induce growth or fecundity, by a proper or improper diet. The effect upon maternal nutrition and in turn upon the offspring have been demonstrated beyond question (24). The need of milk and of the leaves as well as the seeds of vegetation for a full dietary requirement is pointed out. McCollum's water soluble B. and fat-soluble A. are most important factors in this connection. The dental effects of a correct dietary requirement remain to be more fully studied. The dietary effect can be seen in the loosening teeth of the experimental scurvy. All our knowledge of caries points to an unbalanced diet, perhaps a deficient diet together with other factors. Pemberton has noted the effect in other low grade infective processes and McCollum in his experiment has noticed and commented upon the same thing.

I should like to speak of tartar formation in this connection, for I believe it is fully as much a constitutional matter as a local one, but the space for this is too limited. Dr. Black's experiment of fastening a cover glass to his teeth and noting that with over feeding he obtained a deposit upon it demonstrates a step in this same direction. I have spoken of the effect of nutritional balance (25) upon the character of the saliva elsewhere as well as upon the character of tartar and its mode of formation. (26).

We know that as a rule pyorrhea is a disease of the adult. We know that house-fed dogs have it extensively. We know that it accompanies certain diseases or irregular metabolic states, such as diabetes, alcoholism, syphilis. One of the heads of one of our large hospitals has said, "that all his patients have it." It follows disturbances of the glands of internal secretion. It follows chemical and mechanical trauma. As a rule oral conditions are directly dependable upon an infinite number of systemic changes. This is the undeveloped field for study and of the greatest importance to the student of the pathology of the mouth. Anyone can extract teeth but to save them according to the accepted surgical teachings of the day is our problem. Remove all diseased processes surgically. Endeavor to keep up the resistance of the tissues by every known method. Recognize pyorrhea early. Attack the disturbance in its initial stages. Stay the teeth during treatment if necessary. Remedy irregular articulation. Replace missing teeth. Observe all hygienic measures bodily as well as local and the treatment of pyorrhea will be surcessful.

\section{TABLE.}

1. Bass and Johns, Jour. A. M. A., Vol. LIXIV, No. 7 .

2. Howe, Percy R., Dental Cosmos, April, 1916.

3. Craig, Chas, F., Jour. Med. Res., Vol. XXXV, No. 3.

4. Hecker, F., Am. Jour. Med. Science, Vol. CXLIX, No: 6 .

5. Noguchi, Jour. Expt. Med. Vol. XV, No. 1,

6. Drew and Griffin, Jour. of the Royal Microscopied Soc., April, 191-.

7. Howe and Hatch, Jour. Med. Res., Microscopied So., XXXVI, No. 3.

8. Avery, Chickering, Cole, and Dochez-Monograph, No. 7, Rockefeller Institution for Med. Res.

9. Goadby, The Mycology of the Mouth.

10. Hartzell, Jour. Nat. Dental Ass'n.

11. Miller, The Micro-organisms of the Human Mouth.

12. Dick, Geo. F., Jour. Inf. Dis., May, 1918.

13. Worster, Drought-Cecil, British Dent. Jour., Vol. XXXIX, No. 12.

14. Gibbs, J. H., Edinburg Med. Jour., 1917, No. 19.

15. Frances G. Blake, Jour. Expt.-Wed., Vol. XXIV, p. 315.

16. Katherine Howell, Jour. Inf. Dis:, March, 1918, Vol. 22.

17. Nicol, Jour. of M. A., 1914. 
18. Heinemann and Gatewood, Jour. Inf. Dis., $1912, \times 416$.

19. Wright and White, U. S. N. Med. Record, March, 1915.

20. Jackson, Leila, Jour. Inf. Dis., May, 1918.

21. Pitz, W., Jour. Bio. Chem., Vol. XXXI, No. 3.

22. Pemberton, Am. Jour. Med. Science, Vol. ('II, No. 3

23. Osborne and Mendall, Jour. Bio. Chem., Vol. XXXIV, No. 1.

24. MeCollum and Simmonds, Am. Jour., Phy., June, 1918.

25. Dental Cosmos, 1911, p. 43.

26. Proc. Sixth International .Dental Cong., London, 1914.

27. Hall and Harvey, Jour. Med. Res., Vol. XXXV, No. 3.

22. Jackson and Moody, Jour. Inf. Dis., 1916, No. 19.

14. Cozier, Dental Cosmos, June, 1917.

Discussion.

\section{A. W. Ward, San Francisco, Calif.}

I suppose that you all are as well pleased and highly gratified with this paper of Dr. Howe's as I was; yet there are some things that the Doctor has left us in the dark about; as, for instance, concerning immunities. Of course, that is something no one knows very much about; but the Doctor speaks of the susceptibility of the mouth of the guinea pig to pyorrhea following a certain diet, which would lead us to believe that perhaps the human being might also become infected with pyorrhea, due to a faulty diet. That is a possibility. But to me it is hard to conceive of any one, living as we normally do, where we can choose our own foods getting pyorrhea from a faulty diet.

I have had under my care thousands of cases of pyorrhea and I have never seen a true case of pyorrhea caused by salivary calculus. I have seen deposits of calcareous matter. I am speaking now of a calculus. I have seen such products of salivary calculi on the teeth that they became a mechanical irritant, forcing the tissues away, until they final- ly fell out, but I have never seen a true case of pyorrhea caused by salivary calculus, and I do not think they are. Most of them are caused-I agree with Dr. Howe-by mechanical disturbances.

He speaks of the spirochetes and the fusiform bacilli. Pyorrhea in many cases begins very very early, very often by roughened enamel, allowing a lodgment for bacterial placques containing the bacteria that cause suppuration. Too often I believe dentists all over the world look for pus in pyorrhea. There is not microscopic pus in one case in six of what we call pyorrhea. We understand pyorrhea as a destruction or a breaking down of the entire investing material, of the socket of the tooth. And yet very often this destruction goes on for years with no visible external significance. I mean to say there is no irritation of the gums apparent. There is not any pus. You have your patient under your charge all the time.

Some day an infection by a pyogenic micro-organism takes place, and the patient, to your astonishment, comes in, displaying a great deal of pus in the mouth, and you say that this patient got a case of pyorrhea since you saw him last, when a radiograph of the mouth would have shown that the investing material had been breaking down for years, unknown to you.

speaking of the mercurial treatment that we recognize, the deep injection of the succinimate of mercury into the glu. teal muscles, it is hard for me to understand in this day how a thing of that sort could be recommended. The very thing in syphilitic cases that causes pyorrhea or pyorrheal conditions is not the spirochetes. It is the mercury that has been given along with the treatment for the condition.

There is one thing more. It seems so odd to me, when the Doctor speaks of these hospitals filled with patients suffering from arthritis and from all sorts 
of diseases, that any one could conceive in the face of that evidence that these diseases were not caused by the constant absorption of bacteria for years and years, and that it would be expected that as soon as this secondary condition was eradicated, that the patient would get well immediately.

It is not inconceivable when you consider that twenty bacteria can walk abreast on the edge of a razor blade, that a loose tooth, we will take for instance, an upper molar, where the lingual root is entirely denuded of this tissue, where there is always pus present-I said it was not always present but I mean microscopically it is not proved. We know that microscopic pus is present in all of the breaking down of the osseous tissues. The loose teeth act in the way of a syringe, forcing the bacteria directly into the blood stream. Is it not almost inconceivable when we consider as I say that twenty streptococci can walk on the edge of a razor blade, and that these bacteria have been forced back into the blood stream for from five to twenty years, how a scientific man could expect that upon the removal of the teeth the patients would get well immediately. They don't.

The eradication of the foci in the mouth is the biggest step in medicine today. I do not believe that the great advance that is expected from us is going to be brought about by any drugs at all. It is going to be brought about by good surgery, good root surgery, and good bone surgery. You can never hope to inject any sort of a drug into a human being's arm or into their back or anywhere else and remove the seruminal deposit, two-thirds down the length of the root. The only way to do it is to get in and dig, and learn to do good root surgery. (Applause.)

\section{H. J. Leonard, Minneapolis, Minn.}

Dr. Howe's paper fills a long felt want in the hearts of many of us at the University of Minnesota. In the clinic on pyorrhea, we have been brought up on the theory of streptococcus invasion, and that has not answered all of our difficulties. We have often felt that streptococcus invasion was a secondary consideration, and that cleaning up the root surfaces and treating the gums by massage was merely treating symptoms and getting only at secondary conditions.

This consideration of diet, putting the patients health on the standard of proper hygiene, seems to me to be a fundamental consideration in the treatment of pyorrhea, and the quicker we can get ahead of disease of the gums as well as the sequelae and concommitant conditions in the joints and elsewhere, by putting our patients on a proper hygienic dietary regimen with proper exercises, the quicker our patients will be freed from constantly recurring gum and periodontal inflammations.

But there are many other factors, such as loss of teeth, with the subsequent malarticulation that occurs from the shifting of the teeth, that have a great deal to do with the degenerative conditions of the pericementum. It is the belief of many of us who have studied the proposition at Minnesota that the invasion of the gum and periodontal tissues by the streptococcus is due to predisposing causes from systemic mal-metabolism, and local injury from mal-occlusion and malarticulation.

Dr. Howe, closing the discussion: I am very much pleased with what the doctors have said. I heartily agree with it, and if you have noticed carefully what I said, you will see that I advised the very method that the doctor from the West has advocated. I said, "Use every known method of hygiene, local and bodily." Is there any objection to remembering the fact that we have 27 feet of intestine and 5 inches of mouth, and not 27 feet of mouth and 5 inches of intestine? The intestine is a large absorptive tract; we do not want to forget that. 
The tissues of the mouth are tough and fibrous.

I cannot go into a discussion of the foci of infection theory at this time and do it justice, but I will say one thing; the doctor said I did not mention immunity. I did not suppose I would be misunderstood on that. The foci of infection theory takes no account of immunity. Man becomes immune to the natural flora of the mouth, and he is immune to his own flora. A horse from the west, east has to become acclimated. He becomes immune to his new environment. You heard General Gorgas tell last night about his being immune to yellow fever, and you have heard about injecting vaccines to make men immune to various things.

The streptococcus theory does not seem to work out properly on a immunological basis. There is something the matter. This is still a subject for broad study.

It is all right for us to proceed on the faci of infection theory and clean up everything, but don't lie down exclusively on that small and limited viewpoint. The human organism with which we are dealing is a very complicated thing and many physiological and biological processes must be considered. I believe the dental profession is going to become a part of the great medical organization. We need to learn a little more about general conditions, before we begin to learn the specific things.

I want to say one more thing. I recognize that I am dealing with animals. I know of course that if you inject an animal in the ear you do not get pyorrhea in the ear. Rabbits are susceptible to streptococci, but cats are not. When I speak of the spirochetes, I am only speaking of a class of organisms, not of the pallida, which is the cause of syphilis; a dose of succinimate of mercury in certain cases has a marked effect-I do not advocate it as a rule but $I$ have seen pronounced results in certain cases. Diagnose pyorrhea early, use surgical means, keep the parts clean, do all you can to keep up the resistance of the tissues, and you are doing much for your patient. 